\title{
Headache service quality: the role of specialized headache centres within structured headache services, and suggested standards and criteria as centres of excellence
}

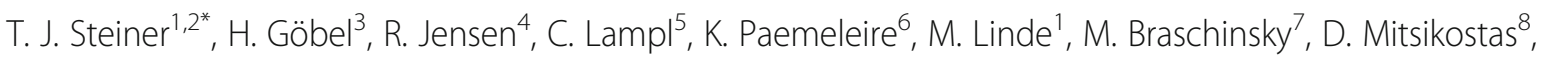 \\ R. Gil-Gouveia ${ }^{9}$ Z. Katsarava ${ }^{10,11,12,13}$ and on behalf of the European Headache Federation and Lifting The Burden: \\ the Global Campaign against Headache
}

\begin{abstract}
In joint initiatives, the European Headache Federation and Lifting The Burden have described a model of structured headache services (with their basis in primary care), defined service quality in this context, and developed practical methods for its evaluation.

Here, in a continuation of the service quality evaluation programme, we set out ten suggested role- and performance-defining standards for specialized headache centres operating as an integral component of these services. Verifiable criteria for evaluation accompany each standard. The purposes are five-fold: (i) to inspire and promote, or stimulate the establishment of, specialized headache centres as centres of excellence; (ii) to define the role of such centres within optimally structured and organized national headache services; (iii) to set out criteria by which such centres may be recognized as exemplary in their fulfilment of this role; (iv) to provide the basis for, and to initiate and motivate, collaboration and networking between such centres both nationally and internationally; ( $v$ ) ultimately to improve the delivery and quality of health care for headache.
\end{abstract}

Keywords: Headache, Health care, Health service organization, Structured headache services, Specialized headache centres, Service quality, Standards, European Headache Federation, Global Campaign against Headache

\section{Introduction}

Headache services must provide health care to very large numbers of people [1], whose illnesses are the secondhighest cause of disability worldwide $[2,3]$. At the same time, unless they aspire to high quality in this purpose, such services are likely to be not only inefficient and wasteful of resources but also ineffectual.

In a joint initiative, the European Headache Federation (EHF) and Lifting The Burden (LTB) have clarified what

\footnotetext{
*Correspondence: t.steiner@imperial.ac.uk

'Department of Neuromedicine and Movement Science, Norwegian University of Science and Technology, Edvard Griegs Gate, Trondheim, Norway

${ }^{2}$ Division of Brain Sciences, Imperial College London, London, UK Full list of author information is available at the end of the article
}

this means, defining "headache service quality" $[4,5]$ and developing methods for its measurement (service quality evaluation: SQE) [6]. These organizations have also described a headache services model - structured, with their base in primary care but with an important and specific role allotted to specialized headache centres [1].

In many countries, however, this role is poorly distinguished: headache centres exist but, performing outside the role boundaries, fail to fulfil it. Here we set out suggested role- and performance-defining standards for specialized headache centres. In doing so, we stress that it is not part of our purpose to set criteria for quality of clinical care. These are properly determined at a national level, and our proposals assume that they are met. Our

(c) The Author(s). 2019 Open Access This article is distributed under the terms of the Creative Commons Attribution 4.0 International License (http://creativecommons.org/licenses/by/4.0/), which permits unrestricted use, distribution, and 
focus is on recommendations for service organization, service quality, professional education and research endeavour that specialized headache centres might follow. Beyond these, we suggest criteria by which specialized headache centres might be generally recognized, nationally and/or internationally, as centres of excellence in the headache community.

We also make clear that neither EHF nor LTB offers itself as a certifying agency, providing accreditation for centres judged to fulfil these criteria. National authorities and other competent agencies might nonetheless, and we hope they will, view the standards set out here as a sound basis for accreditation.

Accordingly, the initiative has five purposes:

- to inspire and promote, or stimulate the establishment of, specialized headache centres as centres of excellence;

- to define the role of such centres within optimally structured and organized national headache services;

- to set out criteria by which such centres may be recognized as exemplary in their fulfilment of this role;

- to provide the basis for, and to initiate and motivate, collaboration and networking between such centres both nationally and internationally;

- ultimately to improve the delivery and quality of health care for headache.

\section{Background: the need for structured headache services}

Headache disorders, especially migraine and tension-type headache (TTH), are common and collectively cause substantial levels of public ill health and disability $[2,7,8]$. Yet, throughout the world, they are under-recognized [9]. This misperception is not easily explained, but it is now slowly changing. On the initiative of LTB and the Global Campaign against Headache [10-13], new studies are filling the gaps in our knowledge of the burdens attributable to headache disorders [14-25], which had embraced half the world [7]. These studies confirm, in all regions of the world, that these burdens weigh heavily not only on people with headache but also on their families, friends, work colleagues and, ultimately, society itself. The Atlas of Headache Disorders published by the World Health Organization (WHO) in collaboration with LTB, although not a population-based survey, collates corroborative evidence on the impact of headache from over 100 countries [9]. The Global Burden of Disease study has found not only that TTH and migraine are respectively the second and third most prevalent disorders in the world [26] but also that migraine is the second most disabling $[2,3]$.

The recognizable consequences not only of public ill health but also of high socioeconomic cost [8], coupled with the large numbers of people affected by headache disorders, give rise to the need for organized, structured and adequately-resourced health services to alleviate them [1, 9, 27]. Effective and cost-effective treatments exist for most people with headache [28]; however, they often fail to reach those who need them [9, 29]. Delivering these treatments is, from any sensible perspective, a public-health priority $[3,9]$. The indirect costs of headache, arising mostly from lost productivity secondary to disability, vastly outweigh direct treatment costs [8]; consequently, from a societal perspective, headacheuntreated costs a great deal more than headache-treated [9]. Even if importance is not attached to the individual burdens attributable to headache [30], society should wish to mitigate the huge financial burden upon itself which headache imposes [9].

Yet, fully developed headache services consume significant health-care resources, and this calls loudly for built-in efficiency with close attention to cost-effectiveness [4-6]. How headache services should be organized with these essentials in mind has been addressed by EHF and LTB in an earlier collaboration $[1,27]$.

\section{Organization of headache services}

While headache disorders are prevalent and ubiquitous, they manifest extremely variably: at one end of the spectrum is mild episodic TTH occurring a few times a year; at the other are highly disabling disorders such as cluster headache and chronic migraine. Not everyone with headache will benefit from, or therefore needs, the same level of care: for this reason, a stratified system is necessary in which, for equity as well as efficiency, specialized care is reserved for and thereby kept available to those who need it.

The three-tier service-organization model of EHF and LTB [1] is summarized below. National modifications may be demanded to align with existing health-care systems and according to resources, but the model has considerable flexibility that allows adaptation without altering its intrinsic structure.

\section{Level 1. General primary care}

Primary care should be the accessible front line for almost all people with headache disorders. At this level, non-specialists - with some training in headache should meet the needs of the great majority of people consulting for headache [1], controlling flow to higher levels.

At level 1, most cases of migraine and TTH should be competently diagnosed and managed [1]. Cluster headache, medication-overuse headache $(\mathrm{MOH})$ and some other common secondary headache disorders should be recognized but not necessarily managed; red-flag warnings of serious secondary headaches should also be 
recognized and duly acted upon. Referral channels to levels 2 and 3, urgent when necessary, should be in place for these cases, and for patients who are diagnostically complex or difficult to manage [1].

This level should also continue long-term care of patients discharged with treatment plans from levels 2 or 3 [1].

\section{Level 2. Special-interest headache care}

Level 2 may, in some countries, be in primary care, provided by general practitioners with a special interest and additional training. In others it is more likely to be offered in polyclinics or district hospitals by neurologists, also with training in headache [1]. Physicians at this level should provide more skilled ambulatory care to most patients referred upwards from level 1 [1].

Their competence should embrace the diagnosis and management of more difficult cases of primary headache and some secondary headache disorders, but not those that are very rare [1]. To fulfil their role, they need access to other services such as neuroimaging, psychology and physiotherapy. For a minority of their patients (perhaps $1 \%$ of all headache patients [1]), they require a referral channel to level 3 (Table 1).

\section{Level 3. Specialized headache centres}

Specialized headache centres are recommended as tertiary referral centres, providing specialist care to patients with primary or secondary headache disorders that are difficult to diagnose or treat, refractory or rare, or for other reasons require specialist intervention (Table 1 ) [1, 31-36]. Patients at level 3 should be a very small subset of patients first seen at level 1 and referred upwards, either via level 2 or directly (and urgently when necessary) [1]; additionally, a few may come from the emergency room.

Table 1 Patients likely to be referred to level 3 (adapted from [1])

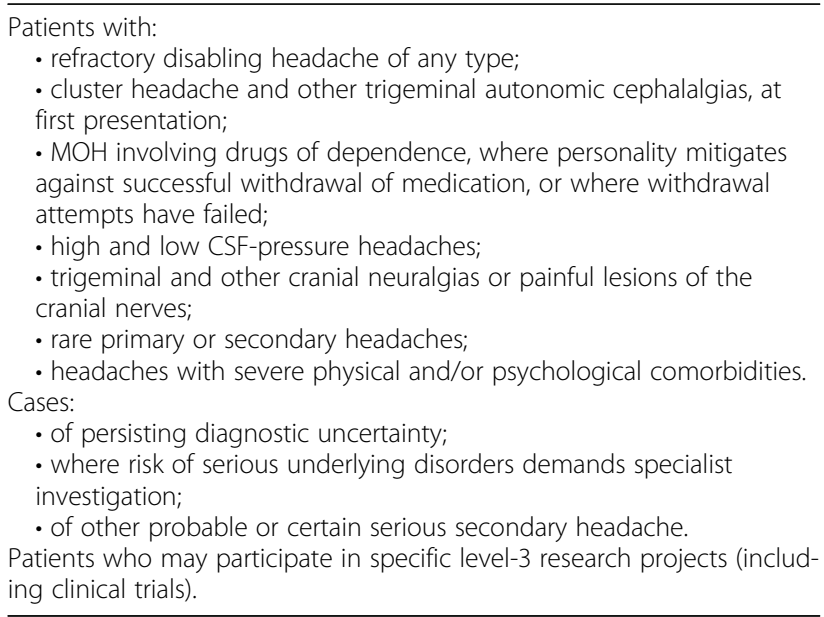

Centres at this level should be nationally-recognized centres of excellence for care, education and research within the headache field; they should concentrate experience in rare primary or secondary headache disorders and cranial neuralgias, and be innovators and/or early adopters of new technologies. They should employ headache specialists and/or neurologists (in either case accredited, when a national accreditation system exists), and be within or closely affiliated to a university or other major hospital with formal academic links [1]. They should offer 24-h inpatient facilities, and have multidisciplinary management competencies. Access to specialists in all other medical fields should provide for the diagnosis and management of the underlying causes of all secondary headache disorders [1].

\section{The role of specialized headache centres}

It is self-evident that specialized headache centres have a role within structured headache services (see Table 2). Nevertheless, in a world with limited resources, and one in which headache wrongly but stubbornly has low priority among calls on these resources [9], this role needs both definition and quantification. Too few specialized centres would not meet need; too many would consume resources that would be better (ie, more cost-effectively) spent at lower levels.

Provided that levels 1 and 2 are adequately set up also, demand at level 3 (Table 1) should be limited to a very small minority of all people needing health care for headache $[1,27]$. It should also be self-regulating, since levels 1 and 2, if adequately set up, will have shorter waiting lists. In reality, headache services are not well structured in most countries [9, 27], and evolution towards better organization is slow. Change requires an evidence base, and few centres have justified their existence by documenting their activities and outcomes in pursuit of their role [31-35, 37-39]. Efficient achievement of desired outcomes is what justifies investment, so demonstration of this is a requirement not just for the continued existence of established specialized centres but also for appropriate expansion in their number.

This is the context in which the need arises for standards for specialized headache centres: standards that

Table 2 The role of specialized headache centres within structured headache services

- to provide best possible level-3 clinical care for adults and/or children, having regard to the resources locally available;

- to support levels 1 and 2 through medical advice;

- to provide training in headache to health-care practitioners at all levels;

- to contribute to the development and/or periodic review and updating

of national management guidelines;

- to conduct research into headache of international value and/or

appropriate to the needs of the local community;

- to provide empirical evidence in support and justification of their existence. 
are universally applicable despite variations in healthcare systems among nations, and maintained by international collaborative networks between centres - themselves made possible by adoption of these standards.

\section{Standards and criteria}

The purpose of standards is to encourage excellence. Criteria are the yardstick by which excellence may be recognized. Criteria, therefore, must be verifiable.

They must also be pragmatic rather than absolute. In less-well-resourced countries, ideals may not be achievable, yet performance backed by aspiration may nonetheless be meritorious in the context, and highly worthy of encouragement.

Table 3 sets out a suggested template for centre evaluation against these standards. It sets out aspirational targets: no benchmarks are proposed, because it is not yet clear what these should be.

\section{Competence of staff} Standard 1

A centre of excellence is staffed by headache specialists, who are sufficient but not excessive in number.

\section{Criteria}

1. A "headache specialist" should be able to document (a) advanced training and (b) past and continuing experience in the field of headache. These are objectively verifiable.

National certification in neurology is not sufficient. Requisite expertise may be self-evident in those with long experience in headache care, whose status and credentials are widely acknowledged and who may be national or international leaders in the field. Otherwise, it may be acquired through recognized training: for example, through full-time attachment for one year to a level-3 centre, or specific training programmes such as the Master Degree courses at Sapienza University of Rome [40-42] or the Danish Headache Centre, University of Copenhagen [43].

2. Sufficiency in number is an obvious requirement for effective operation, whereas excess (in practice unlikely) is wasteful of resources.

What constitutes sufficiency without excess must be determined locally in accordance with how services are organized [1]. This part of the standard is aspirational. In a world of limited resources, a centre may still be recognized as exemplary despite that it is struggling against an excessive workload.

\section{Provision of care Standard 2}

A centre of excellence provides dedicated care for headache patients.

\section{Criterion}

Patients with headache are, as a rule, seen in dedicated sessions, not within general neurological or other sessions. This is objectively verifiable.

\section{Standard 3}

A centre of excellence provides patients with a clear diagnosis made at the earliest opportunity, information about their headache(s), advice on management and internationally-accepted evidence-based treatment.

\section{Criteria}

1. Diagnoses are always according to the International Classification of Headache Disorders (ICHD) (ICHD-3 [44], the latest edition, aligns with WHO's International Classification of Diseases, 11th revision [ICD-11] [45]). This criterion is objectively verifiable.

2. The centre has available and routinely makes use of disability assessments, diagnostic and follow-up diaries, outcome measures and patient information leaflets (such as those published by LTB $[28,46]$ ). This is objectively verifiable.

3. The centre has adopted national or international management guidelines. This is objectively verifiable.

Acceptable national management guidelines are those adopted by national professional organizations or by health authorities. Where none exist, any published international guidelines may be followed (adapted, to the extent necessary, according to availability of treatments).

\section{Standard 4}

A centre of excellence provides full-time multidisciplinary care and (or with access to) round-the-clock inpatient facilities, and competently manages disorders underlying the full range of secondary headaches.

"Full-time" means on a daily basis, not necessarily $24 \mathrm{~h}$ per day, seven days per week.

Capacity in all of these should be sufficient, in the local conditions, to obviate undue treatment delays, but this again is aspirational.

\section{Criteria}

1. Multidisciplinary care is provided through working collaborations between physicians, nurses, physical 
Table 3 Suggested assessment template

\begin{tabular}{llll}
\hline Standard & Criterion & Verification & Target \\
\hline Domain A. Competence of staff & & & \\
1 Centre is staffed by headache & Each "headache specialist" can document: & (a, b) internal audit of CVs and & (a) 100\% yes \\
specialists, who are sufficient & (a) advanced training (yes/no); (b) past and & continuing professional development & (b) 100\% yes \\
but not excessive in number. & continuing experience in the field of & records; & (c) aspirational \\
& headache (yes/no). & (c, d) internal audit of workload and & (d) yes \\
& Number of specialists is: (c) sufficient & waiting times &
\end{tabular}

Domain B. Provision of care

2 Centre provides dedicated care for headache patients.

3 Centre provides patients with a clear diagnosis made at earliest opportunity, information about their headache(s), advice on management and internationallyaccepted evidence-based treatment.

4 Centre provides multidisciplinary care full-time, and competently manages disorders underlying the full range of secondary headaches.
Domain C. Quality evaluation and assurance

5 Centre monitors quality of care in order to optimize it. (yes/no); (d) not excessive (yes/no).

Patients with headache are seen in dedicated sessions, not within general neurological or other sessions (yes/no)

(a) Diagnoses are always according to ICHD-3 (yes/no).

Disability assessments, diagnostic and follow-up diaries, outcome measures and patient information leaflets are: (b) all available (yes/no): (c) all routinely used (yes/no).

(d) National or international management guidelines are adopted (yes/no).

(a) Working collaborations exist between physicians, nurses, physical therapists and psychologists (yes/no).

(b) The centre is based within, or in geographical proximity to, a general hospital providing access to emergency department, neurology, neuroradiology, neurosurgery, psychiatry, ophthalmology, otorhinolaryngology, orthopaedics, rheumatology, cardiology, infectious diseases, endocrinology, paediatrics, gynaecology, dentistry (yes/no).

(c) Inpatient facilities are available for patients with certain comorbidities and for those needing supervised withdrawal from medication overuse (yes/no).

Procedures are in place for recording clinical outcomes and adverse events, and service quality indicators (Table 4), with regular audits of all (yes/no).

Domain D. Networks and collaborations

6 Centre maintains quality of endeavour through networking, collaboration and the sharing of experience with other international and/or national centres.

(a) Existence and operation of networks and collaboration are documented by the centre (yes/no).

(b) Evidence is presented of any of the

following (yes/no):

- exchange of ideas relating to service organization, patient care, teaching and/or research;

- exchange of staff and/or engagement in a

fellowship exchange programme;

- collaborative research protocols;

- shared or collaborative educational

programmes;

- shared or common database.

\section{Domain E. Teaching}

7 Centre is a principal resource for national postgraduate training in the field of headache.
Evidence is presented of recent or current engagement in at least two of the following (yes/no):

- development of national management guidelines, or adaptation of international guidelines for national use; objectively verifiable as present and happening
(a) internal audit of patients' records;
(b) objectively verifiable;
(a) $100 \%$ yes
(c) internal audit of patients' records:
(b) yes
(d) objectively verifiable
(d) yes

(a) existence is objectively verifiable;

(b) access is objectively verifiable;

(a) yes

(c) availability is objectively verifiable

(b) yes

(c) yes (a) yes

(b) yes to one or more 
Table 3 Suggested assessment template (Continued)

\begin{tabular}{|c|c|c|c|c|}
\hline \multicolumn{2}{|c|}{ Standard } & \multirow[b]{2}{*}{$\begin{array}{l}\text { Criterion } \\
\text { - development of learning materials for } \\
\text { trainee headache specialists, neurologists } \\
\text { and/or specialist nurses; } \\
\text { - delivery of didactic teaching and/or clinical } \\
\text { demonstrations to trainee headache } \\
\text { specialists, neurologists and/or specialist } \\
\text { nurses on a regular basis; } \\
\text { - acceptance of clinical trainees on accredited } \\
\text { attachments. }\end{array}$} & \multirow[t]{2}{*}{ Verification } & \multirow[t]{2}{*}{ Target } \\
\hline & & & & \\
\hline 8 & $\begin{array}{l}\text { Centre provides support, through } \\
\text { training and education, to health- } \\
\text { care providers at levels } 1 \text { and } 2 \text {. }\end{array}$ & $\begin{array}{l}\text { Either: } \\
\text { (a) a programme of training and education is } \\
\text { offered through formal links between the } \\
\text { centre and health-care providers at levels } 1 \\
\text { and } 2 \text { throughout the geographical area } \\
\text { served by the centre (yes/no); } \\
\text { (b) where levels } 1 \text { and } 2 \text { are not in place } \\
\text { within structured services, a programme of } \\
\text { training and education is continuously } \\
\text { available to local general practitioners, } \\
\text { nurses and/or pharmacists (yes/no) }\end{array}$ & $\begin{array}{l}\text { (a) objectively verifiable as in place; } \\
\text { (b) verifiable by peer review }\end{array}$ & yes to either \\
\hline \multicolumn{5}{|c|}{ Domain F. Research } \\
\hline 9 & $\begin{array}{l}\text { Centre is a principal fount of useful } \\
\text { research output in the field of } \\
\text { headache. }\end{array}$ & $\begin{array}{l}\text { Research is either or both: } \\
\text { (a) of international value (yes/no); } \\
\text { (b) appropriate to the needs of the local } \\
\text { community (yes/no). }\end{array}$ & $\begin{array}{l}\text { peer review of quantity, quality } \\
\text { and value of publications }\end{array}$ & yes to either \\
\hline \multicolumn{5}{|c|}{ Domain G. Empirical support of existence } \\
\hline 10 & $\begin{array}{l}\text { Centre supports and justifies its } \\
\text { existence, and the development of } \\
\text { others, by documenting and } \\
\text { demonstrating its utility. }\end{array}$ & $\begin{array}{l}\text { Activities and achievements: } \\
\text { (a) are documented (yes/no); } \\
\text { (b) provide evidence of utility (yes/no). }\end{array}$ & $\begin{array}{l}\text { (a) objectively verifiable; } \\
\text { (b) verifiable by peer review }\end{array}$ & $\begin{array}{l}\text { (a) yes } \\
\text { (b) yes }\end{array}$ \\
\hline
\end{tabular}

therapists and psychologists. Existence of these collaborations is objectively verifiable.

Physical therapists and psychologists collaborating with the service need not be employed within it.

2. There is access to a full range of other specialists. This is objectively verifiable.

Accessible specialties should include at least the following: neurology, neuroradiology, neurosurgery, psychiatry, ophthalmology, otorhinolaryngology, orthopaedics, rheumatology, cardiology, infectious diseases, endocrinology, paediatrics, gynaecology and dentistry. This requirement implies that the centre is within or works very closely with and in geographical proximity to a general hospital offering an emergency department.

3. Inpatient facilities, on-site or nearby, are accessible for the care and management of patients with certain comorbidities, and for those needing supervised withdrawal from medication overuse. This is objectively verifiable.

\section{Quality evaluation and assurance Standard 5}

A centre of excellence monitors quality of care in order to optimize it.

\section{Criterion}

Procedures are in place for recording clinical outcomes and adverse events, and service quality indicators according to Table 4 [5]. All are audited regularly as part of quality assurance. These are objectively verifiable as present and happening, or not.

\section{Networks and collaborations \\ Standard 6}

Centres of excellence maintain quality of endeavour through networking, collaboration and the sharing of experience with other internationally- and/or nationallyrecognized centres.

The nationwide German headache treatment network provides good examples of networking and collaboration at national [33, 34] and international [36] levels.

\section{Criteria}

1. The existence and operation of networks and collaboration are documented by the centre. This is verifiable by peer review.

2. Evidence is presented of any or all of the following: - exchange of ideas relating to service organization, patient care, teaching and/or research; 
Table 4 Domains and indicators of quality in headache service delivery (from [5])

Domain A. Accurate diagnosis is essential for optimal headache care

A1 Patients are asked about onset of their headaches

A2 Diagnosis is according to current ICHD criteria

A3 A working diagnosis is made at the first visit

A4 A definitive diagnosis is made at first or subsequent visit

A5 Diagnosis is reviewed during later follow-up

A6 Diaries are used to support or confirm diagnosis

Domain B. Individualized management is essential for optimal headache care

B1 Waiting-list times for appointments are related to urgency of need

B2 Sufficient time is allocated to each visit for the purpose of good management

B3 Patients are asked about the temporal profile of their headaches

B4 Treatment plans follow evidence-based guidelines, reflecting diagnosis

B5 Treatment plans include psychological approaches to therapy when appropriate

B6 Treatment plans reflect disability assessment

B7 Patients are followed up to ascertain optimal outcome

Domain C. Appropriate referral pathways are essential for optimal headache care

C1 Referral pathway is available from primary to specialist care

C2 Urgent referral pathway is available when necessary

Domain D. Education of patients about their headaches and their management is essential for optimal headache care

D1 Patients are given the information they need to understand their headache and its management

D2 Patients are given appropriate reassurance

Domain E. Convenience and comfort are part of optimal headache care

E1 The service environment is clean and comfortable

E2 The service is welcoming

E3 Waiting times in the clinic are acceptable to both health-care providers and patients

Domain F. Achieving patient satisfaction is part of optimal headache care

F1 Patients are satisfied with their management

Domain G. Optimal headache care is efficient and equitable

G1 Procedures are followed to ensure resources are not wasted

G2 Patients are not over-investigated

G3 Costs of the service are measured as part of a cost-effectiveness policy

G4 There is equal access to headache services for all who need it

Domain $\mathrm{H}$. Outcome assessment is essential in optimal headache care

H1 Outcome measures are based on self-reported symptom burden (headache frequency, duration and intensity)

H2 Outcome measures are based on self-reported disability burden

H3 Outcome measures are based on self-reported quality of life

Domain I. Optimal headache care is safe

11 Patients are not over-treated

12 Systems are in place to be aware of serious adverse events
- exchange of staff and/or engagement in a fellowship exchange programme;

- collaborative research protocols;

- shared or collaborative educational programmes;

- shared or common database.

These are verifiable by peer review.

\section{Teaching}

\section{Standard 7}

Centres of excellence are a principal resource for national postgraduate training in the field of headache.

\section{Criterion}

Evidence is presented of recent or current engagement in at least two of the following:

- development of national management guidelines, or adaptation of international guidelines for national use;

- development of learning materials for trainee headache specialists, neurologists and/or specialist nurses;

- delivery of didactic teaching and/or clinical demonstrations to trainee headache specialists, neurologists and/or specialist nurses on a regular basis;

- acceptance of clinical trainees on accredited attachments.

These are verifiable by peer review.

\section{Standard 8}

Centres of excellence delivering level-3 care within structured headache services also provide support, through training and education, to health-care providers at levels 1 and 2.

It is understood that, in some countries or areas, levels 1 and 2 may not be in place.

\section{Criterion}

Either of (a) or (b):

a) Formal links exist between the centre and health-care providers at levels 1 and 2 throughout the geographical area served by the centre. Through these links, a programme of training and education is offered. The existence of these is objectively verifiable.

b) Where levels 1 and 2 are not in place within structured services, a programme of training and education is continuously available to local general practitioners, nurses and/or pharmacists. The existence of this alternative is verifiable by peer review. 


\section{Research}

\section{Standard 9}

Centres of excellence are a principal fount of useful research output in the field of headache.

\section{Criterion}

Research by the centre is of international value and/or appropriate to the needs of the local community. The quantity, quality and value of publications are verifiable by peer review.

Research may include development and maintenance of patient databases, public-health initiatives, epidemiology, pathophysiological and other clinical research, evaluation of diagnostic and therapeutic guidelines and therapeutic trials into which there is intellectual input. Simple recruitment into industry-sponsored trials does not fulfil this criterion.

\section{Empirical support of existence Standard 10}

Centres of excellence support and justify their existence, and the development of others, by documenting their activities and achievements and demonstrating their utility within structured headache services.

\section{Criteria}

1. Activities and achievements are documented. This is objectively verifiable.

2. Activities and achievements provide evidence of utility. This is verifiable by peer review.

\section{Conclusions}

Ten standards are defined, each one accompanied by one or more verifiable criteria. Collectively these define the role of specialized headache centres within structured headache services, and allow for evaluation of performance as part of service-quality assurance. Their adoption should, ultimately, improve the delivery and quality of health care for headache.

Agencies with appropriate competence and authority might use these standards as a basis for centreaccreditation.

\section{Abbreviations \\ CSF: Cerebrospinal fluid; EHF: European Headache Federation; ICD: International Classification of Diseases; ICHD: International Classification of Headache Disorders; LTB: Lifting The Burden; $\mathrm{MOH}$ : Medication-overuse headache; SQE: Service-quality evaluation; TTH: Tension-type headache; WHO: World Health Organization}

\section{Acknowledgements}

These standards and criteria were developed as a service-quality project within the Global Campaign against Headache, which is led by the nongovernmental organization Lifting The Burden in official relations with the World Health Organization, and in collaboration with European Headache Federation.
Funding

None

Availability of data and materials

All relevant data are included, or specifically cited, in the manuscript.

\section{Authors' contributions}

TJS, HG and RJ were responsible for conception and first draft of these standards and criteria. All authors reviewed them critically, provided intellectual input, contributed to revision and approved the final version.

Ethics approval and consent to participate

Not applicable.

\section{Consent for publication}

Not applicable.

\section{Competing interests}

TJS, RJ and ZK are Directors and Trustees of Lifting The Burden. CL, KP, ML, MB, DM and ZK are Directors and Trustees of European Headache Federation. Otherwise, no author declares a conflict of interest.

\section{Publisher's Note}

Springer Nature remains neutral with regard to jurisdictional claims in published maps and institutional affiliations.

\section{Author details}

${ }^{1}$ Department of Neuromedicine and Movement Science, Norwegian University of Science and Technology, Edvard Griegs Gate, Trondheim, Norway. ${ }^{2}$ Division of Brain Sciences, Imperial College London, London, UK. ${ }^{3}$ Kiel Headache Centre, Kiel Neurological Pain and Headache Centre, Kiel, Germany. ${ }^{4}$ Danish Headache Centre, Department of Neurology, University of Copenhagen, Rigshospitalet-Glostrup Hospital, Glostrup, Denmark. ${ }^{5}$ Headache Medical Centre, Ordensklinikum Linz Barmherzige Schwestern, Linz, Austria. ${ }^{6}$ Department of Neurology, Ghent University Hospital, Ghent, Belgium. ${ }^{7}$ Headache Clinic, Neurology Clinic, Tartu University Clinics, Tartu, Estonia. ${ }^{8}$ Neurology Department A, Aeginition Hospital, National \& Kapodistrian University of Athens, Athens, Greece. ${ }^{9}$ Hospital da Luz Headache Center, Lisbon, Portugal. ${ }^{10}$ Evangelical Hospital Unna, Unna, Germany. ${ }^{11}$ Department of Neurology, University of Duisburg-Essen, Essen, Germany. ${ }^{12}$ EVEX Medical Corporation, Tbilisi, Georgia. ${ }^{13}$ IM Sechenov First Moscow State Medical University (Sechenov University), Moscow, Russian Federation.

Received: 13 December 2018 Accepted: 11 February 2019 Published online: 04 March 2019

\section{References}

1. Steiner TJ, Antonaci F, Jensen R, Lainez MJ, Lanteri-Minet M, Valade D (2011) Recommendations for headache service organisation and delivery in Europe. J Headache Pain 12:419-426.

2. Vos T, Abajobir AA, Abate KH, Abbafati C, Abbas KM, Abd-Allah F, Abdulkader RS, Abdulle AM, Abebo TA, Abera SF, Aboyans V, Abu-Raddad L, Ackerman IN, Adamu AA, Adetokunboh O, Afarideh M, Afshin A, Agarwal SK, Aggarwal R, Agrawal A, Agrawal S, Ahmadieh H, Ahmed MB, Aichour MTE, Aichour AN, Aichour I, Aiyar S, Akinyemi RO, Akseer N, Al Lami FH, Alahdab F, Al-Aly Z, Alam K, Alam N, Alam T et al (2017) Global, regional, and national incidence, prevalence, and years lived with disability for 328 diseases and injuries for 195 countries, 1990-2016: a systematic analysis for the global burden of disease study 2016. Lancet 390:1211-1259.

3. Steiner TJ, Stovner LJ, Vos T, Jensen R, Katsarava Z (2018) Migraine is first cause of disability in under 50s: will health politicians now take notice? (editorial). J Headache Pain 19:17.

4. Peters M, Perera S, Loder E, Jenkinson C, Gil Gouveia R, Jensen R, Katsarava Z, Steiner TJ (2012) Quality in the provision of headache care. 1: systematic review of the literature and commentary. J Headache Pain 13:437-447.

5. Peters M, Jenkinson C, Perera S, Loder E, Jensen R, Katsarava Z, Gil Gouveia R, Broner S, Steiner T (2012) Quality in the provision of headache care. 2: defining quality and its indicators. J Headache Pain 13:449-457.

6. Schramm S, Uluduz D, Gil Gouveia R, Jensen R, Siva A, Uygunoglu U, Gvantsa G, Mania M, Braschinsky M, Filatova E, Latysheva N, Osipova V, Skorobogatykh K, Azimova J, Straube A, Emre Eren O, Martelletti P, De 
Angelis V, Negro A, Linde M, Hagen K, Radojicic A, Zidverc-Trajkovic J, Podgorac A, Paemeleire K, De Pue A, Lampl C, Steiner TJ, Katsarava Z (2016) Headache service quality: evaluation of quality indicators in 14 specialistcare centres. J Headache Pain 17:111.

7. Stovner LJ, Hagen K, Jensen R, Katsarava Z, Lipton RB, Scher Al, Steiner TJ, Zwart J-A (2007) The global burden of headache: a documentation of headache prevalence and disability worldwide. Cephalalgia 27:193-210.

8. Linde M, Gustavsson A, Stovner LJ, Steiner TJ, Barré J, Katsarava Z, Lainez JM, Lampl C, Lantéri-Minet M, Rastenyte D, Ruiz de la Torre E, Tassorelli C, Andrée C (2012) The cost of headache disorders in Europe: the Eurolight project. Eur J Neurol 19:703-711.

9. World Health Organization and Lifting The Burden (2011) Atlas of headache disorders and resources in the world 2011. WHO, Geneva at: http://www. who.int/mental_health/management/atlas_headache_disorders/en/2011.

10. Steiner TJ (2004) Lifting the burden: the global campaign against headache. Lancet Neurol 3:204-205.

11. Steiner TJ (2005) Lifting The Burden: the global campaign to reduce the burden of headache worldwide. J Headache Pain 6:373-377.

12. Steiner TJ, Birbeck GL, Jensen R, Katsarava Z, Martelletti P, Stovner $\sqcup$ (2010) Lifting The Burden: the first 7 years. J Headache Pain 11:451-455.

13. Steiner TJ, Birbeck GL, Jensen R, Katsarava Z, Martelletti P, Stovner $\sqcup$ (2011) The global campaign, World Health Organization and Lifting The Burden: collaboration in action. J Headache Pain 12:273-274.

14. Yu S, Liu R, Zhao G, Yang X, Qiao X, Feng J, Fang Y, Cao X, He M, Steiner T (2012) The prevalence and burden of primary headaches in China: a population-based door-to-door survey. Headache 52:582-591.

15. Ayzenberg I, Katsarava Z, Sborowski A, Chernysh M, Osipova V, Tabeeva G, Yakhno N, Steiner TJ (2012) The prevalence of primary headache disorders in Russia: a countrywide survey. Cephalalgia 32:373-381.

16. Kulkarni GB, Rao GN, Gururaj G, Stovner LJ, Steiner TJ (2015) Headache disorders and public ill-health in India: prevalence estimates in Karnataka state. J Headache Pain 16:67.

17. Rao GN, Kulkarni GB, Gururaj G, Stovner LJ, Steiner TJ (2015) The burden attributable to headache disorders in India: estimates from a communitybased study in Karnataka state. J Headache Pain 16:94.

18. Herekar AA, Ahmad A, Uqaili UL, Ahmed B, Effendi J, Alvi SZ, Shahab MA, Javed U, Herekar AD, Khanani R, Steiner TJ (2017) Primary headache disorders in the adult general population of Pakistan - a cross sectional nationwide prevalence survey. J Headache Pain 18:28.

19. Manandhar K, Risal A, Steiner TJ, Holen A, Linde M (2015) The prevalence of primary headache disorders in Nepal: a nationwide population-based study. $J$ Headache Pain 16:95.

20. Manandhar K, Risal A, Linde M, Steiner TJ (2016) The burden of headache disorders in Nepal: estimates from a population-based survey. J Headache Pain 17:3.

21. Mbewe E, Zairemthiama P, Yeh H-H, Paul R, Birbeck GL, Steiner TJ (2015) The epidemiology of primary headache disorders in Zambia: a populationbased door-to-door survey. J Headache Pain 16:30.

22. Mbewe E, Zairemthiama P, Paul R, Birbeck GL, Steiner TJ (2015) The burden of primary headache disorders in Zambia: national estimates from a population-based door-to-door survey. J Headache Pain 16:36.

23. Zebenigus M, Tekle-Haimanot R, Worku DK, Thomas H, Steiner TJ (2016) The prevalence of primary headache disorders in Ethiopia. J Headache Pain 17:110

24. Zebenigus M, Tekle-Haimanot R, Worku DK, Thomas H, Steiner TJ (2016) The burden of headache disorders in Ethiopia: national estimates from a population-based door-to-door survey. J Headache Pain 18:58.

25. Steiner TJ, Stovner LJ, Katsarava Z, Lainez JM, Lampl C, Lantéri-Minet M, Rastenyte D, Ruiz de la Torre E, Tassorelli C, Barré J, Andrée C (2014) The impact of headache in Europe: principal results of the Eurolight project. J Headache Pain 15:31.

26. Vos T, Flaxman AD, Naghavi M, Lozano R, Michaud C, Ezzati M, Shibuya K, Salomon JA, Abdalla S, Aboyans V, Abraham J, Ackerman I, Aggarwal R, Ahn SY, Ali MK, Alvarado M, Anderson HR, Anderson LM, Andrews KG, Atkinson C, Baddour LM, Bahalim AN, Barker-Collo S, Barrero LH, Bartels DH, Basáñez MG, Baxter A, Bell ML, Benjamin EJ, Bennett D, Bernabé E, Bhalla K, Bhandari B, Bikbov B, Bin Abdulhak A et al (2012) Years lived with disability (YLDs) for 1160 sequelae of 289 diseases and injuries 1990-2010: a systematic analysis for the global burden of disease study 2010. Lancet 380:2163-2196.
27. Antonaci F, Valade D, Lanteri-Minet M, Láinez JM, Jensen R, Steiner TJ (2008) Proposals for the organisation of headache services in Europe. Intern Emerg Med 3(suppl 1):S25-S28.

28. Steiner TJ, Jensen R, Katsarava Z, Linde M, MacGregor EA, Osipova V, Paemeleire $\mathrm{K}$, Olesen J, Peters M, Martelletti $\mathrm{P}$, on behalf of the European Headache Federation and Lifting The Burden: the Global Campaign against Headache (2019) Aids to management of headache disorders in primary care (2nd edition). J Headache Pain 20 (in press).

29. Steiner TJ, Stovner LI, Dua T, Birbeck GL, Jensen R, Katsarava Z, Martelletti $P$, Saxena S (2011) Time to act on headache disorders. J Headache Pain 12: 501-503.

30. Steiner TJ, Gururaj G, Andrée C, Katsarava Z, Ayzenberg I, Yu SY, Al Jumah M, Tekle-Haimanot R, Birbeck GL, Herekar A, Linde M, Mbewe E, Manandhar K, Risal A, Jensen R, Queiroz LP, Scher Al, Wang SJ, Stovner LJ (2014) Diagnosis, prevalence estimation and burden measurement in population surveys of headache: presenting the HARDSHIP questionnaire. J Headache Pain 15:3.

31. Harpole LH, Samsa GP, Jurgelski AE, Shipley JL, Bernstein A, Matchar DB (2003) Headache management program improves outcome for chronic headache. Headache 43:715-724.

32. Lake AE, Saper JR, Hamel RL (2009) Comprehensive inpatient treatment of refractory chronic daily headache. Headache 49:555-562.

33. Göbel H, Heinze A, Heinze-Kuhn K, Henkel K, Roth A, Rüschmann HH (2009) Development and implementation of integrated health care in pain medicine: the nationwide German headache treatment network. Schmerz 23:653-670.

34. Göbel H, Heinze-Kuhn K, Petersen I, Göbel A, Heinze A (2013) Integrated headache care network. Kiel migraine and headache center and German National Headache Treatment Network. Schmerz 27:149-165.

35. Jensen R, Zeeberg P, Dehlendorff C, Olesen J (2010) Predictors of outcome of the treatment programme in a multidisciplinary headache center. Cephalalgia 30:1214-1224.

36. Diener H, Gaul C, Jensen R, Göbel H, Heinze A, Silberstein S (2011) Integrated headache care. Cephalalgia 31:1039-1047.

37. Saper JR, Lake AE, Madden SF, Kreeger C (1999) Comprehensive tertiary care for headache: a 6-month outcome study. Headache 39:249-263.

38. Lemstra M, Stewart B, Olszynski WP (2002) Effectiveness of multidisciplinary intervention in the treatment of migraine: a randomized clinical trial. Headache 42:845-854

39. Rothrock JF, Parada VA, Sims C, Key K, Walters NS, Zweifler RM (2006) The impact of intensive patient education on clinical outcome in a clinic-based migraine population. Headache 46:726-731.

40. Martelletti P, Haimanot RT, Lainez MJA, Rapoport AM, Ravishankar K, Sakai F, Silberstein SD, Vincent M, Steiner TJ (2005) The global campaign to reduce the burden of headache worldwide. The international team for specialist education (ITSE). J Headache Pain 6:261-263.

41. Martelletti P (2007) Headache medicine: academic teaching in Europe. The Sapienza University Master's Degree. Headache 47:1224-1225 author reply 1225-1227.

42. Sapienza University of Rome. Master in Headache Medicine. At: https://web. uniroma1.it/masterheadache/.

43. University of Copenhagen. Master of Headache Disorders. At: https://mhd.ku.dk/.

44. Headache Classification Committee of the International Headache Society (2018) The international classification of headache disorders, 3rd edition. Cephalalgia 38:1-211.

45. World Health Organization (2018) International Classification of Diseases 11th Revision. The global standard for diagnostic health information. WHO, Geneva at https://icd.who.int.

46. Steiner TJ, Paemeleire $K$, Jensen $R$, Valade $D$, Savi L, Lainez MJA, Diener H-C, Martelletti P, Couturier EGM on behalf of the European Headache Federation and Lifting The Burden: The global campaign to reduce the burden of headache worldwide (2007) Aids for management of common headache disorders in primary care. J Headache Pain 8 suppl 1: S22-S39. 